\title{
LE DICTIONNAIRE COMME CHAMP DE LUTTE IDÉOLOGIQUE : LES IDÉES DE RELIGION NATURELLE ET DE RELIGION UNIVERSELLE DANS LES DICTIONNAIRES DU SIÈCLE DES LUMIÈRES (1764-1775)
}

\section{INTRODUCTION}

Le dictionnaire, surtout de caractère encyclopédique ou thématique, du fait de son aspiration à embrasser dans un seul ouvrage la totalité du réel à travers les mots et leurs définitions, se prête particulièrement bien à véhiculer une vision totale, quoique fragmentée, du monde ${ }^{1}$. Cette dimension philosophique et herméneutique du dictionnaire se manifeste tout spécialement au siècle des Lumières ${ }^{2}$, " siècle des dictionnaires " s'il en fut, où la modernité frappe aux portes de l'Ancien régime. C'est dans ce contexte que je me propose d'étudier quelques exemples du conflit idéologique mené dans certains dictionnaires choisis publiés à l'époque (entre 1764 et 1775). La perspective thématique et philosophico-religieuse de la présente étude, relevant dans une large mesure de l'histoire des idées, concerne plus précisément les idées de religion naturelle et de religion universelle,

1 «Chefs-d'œuvre ou compilations médiocres, les dictionnaires nous offrent, comme les œuvres littéraires, les monuments, les institutions et les créations de la mode, une image précieuse d'une certaine civilisation, et ils mériteraient d'être étudiés attentivement par les historiens des idées, non seulement à cause des renseignements qu'ils nous fournissent sur les mots, mais encore, et surtout peut-être, en raison de l'esprit qui a animé leurs auteurs et des conditions qui ont assuré leur diffusion. » (G. Matoré, Histoire des dictionnaires français, Librairie Larousse, Paris 1968, p. 26).

${ }^{2}$ Sur les lexiques et encyclopédies du XVIII ${ }^{\mathrm{e}}$ siècle, voir : ibidem, pp. 88-108. 
en tant que termes conflictuels s'inscrivant dans des visions du monde opposées ou alternatives.

Les temps modernes ont vu la naissance progressive de l'idée moderne de religion, conçue comme ensemble de pratiques fondé sur un système doctrinal. Le Dictionnaire de l'Académie Française (édition de 1694) définit la religion comme «Culte qu'on rend à la Divinité, suivant la creance que 1'on en a » . Selon l'Encyclopédie, la religion n'est autre que « la connoissance de la divinité, et celle du culte qui lui est dû ${ }^{4}$. Cette façon d'envisager la religion est étroitement liée à l'explosion de la Réforme et à l'élargissement de l'horizon religieux à l'époque de la Renaissance, ainsi qu'au développement d'études comparées sur la religion à partir du XVII ${ }^{\mathrm{e}}$ siècle. Elle doit être associée à la conscience croissante de la pluralité des croyances existant dans le monde. Son apparition se verra accompagnée de l'évolution accélérée des idées de religion naturelle et de religion universelle (la première constituant une variante de la seconde).

Les idées en question plongent leurs racines dans un passé lointain. Cicéron, face aux contradictions dans les opinions des différentes sectes philosophiques portant sur la nature des dieux, trouve chez les stoïciens les notions permettant de trouver une base commune et raisonnable des opinions et des croyances, nécessaire à l'exercice du culte public ${ }^{5}$. Au temps de la Renaissance, la religion naturelle constitue un postulat de caractère irénique, favorisant la liberté de conscience, distinguant les points fondamentaux de la foi des questions litigieuses et secondaires ${ }^{6}$. Alors qu'au XVII ${ }^{\mathrm{e}}$ siècle elle est toujours comprise par certains comme la première étape d'un christianisme authentique ou épuré, chez d'autres elle devient un instrument de critique et vise à libérer la religion de toute tutelle ${ }^{7}$, conformément à la tendance qui caractérise la «crise de la conscience européenne » analysée par Paul Hazard ${ }^{8}$. À l'âge classique, la religion naturelle constitue le dénominateur commun de tous les cultes, associé à un credo minimum qui se résume dans quelques points essentiels ${ }^{9}$, et dont la formulation varie selon l'auteur : 1) existence de Dieu et de la Providence ; 2) culte intérieur assimilé à la pratique de la justice ou de la charité ; 3) liberté et responsabilité morale de l'homme ; 4) récompense de la vertu (immanente ou dans la vie future). Le Grand Siècle, et tout

${ }^{3}$ Le dictionnaire de l'Académie françoise, dédié au Roy, 2 vol., Vve J.B. Coignard et J.B. Coignard, Paris 1694, t. 2, <http://gallica.bnf.fr/ark:/12148/bpt6k50398c> [consulté le 29.06.2015], p. 391 (attention à l'erreur de pagination dans l'ouvrage).

${ }^{4}$ Encyclopédie ou Dictionnaire raisonné des sciences, des arts et des métiers, par une société de gens de lettres, 17 vol., Briasson — David - Le Breton, Paris / S. Faulche, Neuchâtel 17511765, t. 14, <http://gallica.bnf.fr/ark:/12148/bpt6k50546p> [consulté le 29.06.2015], pp. 78-88.

${ }^{5}$ J. Lagrée, La religion naturelle, PUF, Paris 1991, pp. 15-25.

${ }^{6}$ Ibidem, pp. 36-40.

${ }^{7}$ Ibidem, p. 41.

${ }^{8}$ P. Hazard, La crise de la conscience européenne (1680-1715), Le Livre de Poche, Paris 1994 (1935) ; voir surtout le chapitre II de la troisième partie « Le déisme et la religion naturelle».

9 J. Lagrée, op. cit., pp. 42-51. 
spécialement sa seconde moitié, avec la diffusion de la pensée d'auteurs comme Spinoza, Locke ou Toland, adoptant tous une certaine idée de la religion naturelle, peut être considéré comme l'aube des Lumières. Le Dictionnaire historique et critique de Bayle, publié en 1697, anticipe à plusieurs égards l'Encyclopédie de Diderot et d'Alembert.

\section{L'ARTICLE «RELIGION» DANS LES DICTIONNAIRES DES TEMPS MODERNES}

L'article « Religion » de l'Encyclopédie (volume 14 publié en 1765) s'ouvre sur la distinction entre religion naturelle et religion révélée. La première, assimilée à la morale ou à l'éthique, est « le culte que la raison, laissée à elle-même, et à ses propres lumières, apprend qu'il faut rendre à l'Être suprême, auteur et conservateur de tous les êtres qui composent le monde sensible $»^{10}$. La seconde est « celle qui nous instruit de nos devoirs envers Dieu, envers les autres hommes, et envers nous-mêmes, par quelques moyens surnaturels $»^{11}$. Curieusement, l'article du chevalier de Jaucourt expose plusieurs arguments en faveur de l'insuffisance de la religion naturelle, sans en fournir aucun en faveur de sa suffisance. Selon lui, la religion naturelle « consiste en l'accomplissement des devoirs qui nous lient à la divinité 》, réduits au nombre de trois : « à l'amour, à la reconnoissance et aux hommages $»^{12}$. Et plus loin l'article ne laisse pas de surprendre de nouveau, en présentant le christianisme comme « la religion Chrétienne, au culte de laquelle tout homme est obligé de se soumettre, parce que c'est la seule véritable, qu'elle a été marquée au sceau de la Divinité », puis en réfutant le relativisme religieux au nom de l'autorité de l'Église, qui réglemente la manière d'exercer le culte divin ${ }^{13}$.

Il est intéressant de comparer l'article « Religion » de l'Encyclopédie avec ceux du Dictionnaire de Trévoux, rédigé par les jésuites à partir de 1704, et dont l'édition de 1771 doit être située dans un contexte de polémique avec les encyclopédistes. La comparaison est d'autant plus intéressante que le Dictionnaire de Trévoux est largement tributaire du Dictionnaire universel de l'abbé Furetière (1690), les jésuites ayant toutefois pris le soin de purger l'original des ajouts effectués par Basnage de Beauval (protestant et auteur d'un ouvrage intitulé Tolérance des religions) dans l'édition de 1701. Le Dictionnaire de Trévoux (6 $6^{\mathrm{e}}$ édition de 1771) reprend plus d'une expression de l'article de Jaucourt, notamment la

\footnotetext{
${ }^{10}$ Encyclopédie..., pp. 78-79.

11 Ibidem, p. 79.

12 Ibidem.

${ }^{13}$ Ibidem, p. 83.
} 
distinction entre religion naturelle et révélée ${ }^{14}$, absente du Dictionnaire universel de Furetière et de celui de Beauval.

La façon de commencer l'article est symptomatique de la visée des éditeurs. Alors que les considérations sur la religion naturelle ouvrent pratiquement l'article de Jaucourt, le Dictionnaire de Trévoux semble plutôt mettre l'accent sur l'attitude de piété associée à la religion. Le mot désignant celle-ci marque « une qualité de l'âme et une disposition du cœur qui fait qu'on ne manque point à ce qu'on doit à l'Être suprême $»^{15}$. De cette façon, les jésuites de Trévoux s'inscrivent dans une tradition ancienne qui voyait dans la religion une vertu et une disposition de l'âme plutôt qu'une forme de culte liée à un ensemble doctrinal, suivant l'acception moderne du terme ${ }^{16}$. Le dictionnaire de Furetière de 1690 définit la religion comme « Culte du vray Dieu, ceremonies exterieures par lesquelles on témoigne qu'on l'adore dans son cœur », en précisant d'emblée que « La vraye Religion est la Catholique, Apostolique et Romaine. Tous les cultes des faux Dieux ne sont que superstition, ne s'appellent Religion qu'abusivement ${ }^{17}$. L'article de l'édition de Basnage, même s'il reprend par la suite la thèse sur les cultes des faux dieux, omet naturellement l'identification de la vraie religion au catholicisme et fait suivre la définition de la religion («Culte qu'on rend à Dieu : sentiment, creance de la divinité ») d'une remarque de tonalité sceptique concernant le rapport entre la foi et la raison : " Il est bien peu en qui la Religion soit le fruit de l'étude, et de la reflexion. Nous sommes plus inquietez que persuadez de la Religion, qui ne tombe point sous l'évidence des sens $»^{18}$.

Cette brève étude comparative de l'article « Religion » dans l'Encyclopédie de Diderot et d'Alembert, le Dictionnaire de Trévoux, ainsi que le Dictionnaire universel de Furetière relayé par celui de Beauval, permet d'établir bien des rapprochements entre les ouvrages. Même si elle met en évidence certaines divergences sur le plan idéologique, c'est surtout entre le Furetière et sa version corrigée par Beauval. On attendrait sans doute une opposition plus tranchée entre le

${ }^{14}$ Dictionnaire universel françois et latin : vulgairement appelé dictionnaire de Trévoux, $6^{\mathrm{e}}$ édition, 8 vol., Compagnie des libraires associés, Paris 1771, t. 7, <http://gallica.bnf.fr/ark:/12148/ bpt6k509861> [consulté le 29.06.2015], p. 259.

15 Ibidem.

${ }^{16}$ Toutefois, l'article de l'Encyclopédie ne néglige pas non plus cet aspect de la religion, notamment en soulignant l'importance des devoirs envers Dieu, la priorité du culte intérieur ou encore la nécessité d'un culte extérieur. Voir : Encyclopédie..., pp. 80-82.

${ }^{17}$ A. Furetière, Dictionnaire universel, contenant généralement tous les mots françois tant vieux que modernes et les termes des sciences et des arts, Arnout et Reinier Leers, La Haye-Rotterdam 1690, t. 3, https://books.google.fr/books?id=rjIbrwy60qcC\&printsec $=$ frontcover\&hl=fr\#v=onepage \& $\mathrm{q} \& \mathrm{f}=$ false, p. 359.

${ }^{18}$ A. Furetière, Dictionnaire universel, contenant généralement tous les mots françois tant vieux que modernes et les termes des sciences et des arts, $2^{\mathrm{e}}$ édition revue et corrigée par Basnage de Beauval, 3 vol., Arnout et Reinier Leers, La Haye 1701, t. 3, <http://gallica.bnf.fr/ark:/12148/ bpt6k56749155> [consulté le 29.06.2015], p. 511. 
dictionnaire jésuite et celui des encyclopédistes, qui par endroits, paraît même plus orthodoxe du point de vue chrétien que le premier, même s'il semble davantage mettre en valeur l'idée de religion naturelle. Mais c'est sur d'autres exemples datant de la même période qu'il est possible d'observer plus clairement le conflit autour des idées religieuses qui bouleverse alors la société.

\section{VOLTAIRE ET L'IDÉE DE RELIGION NATURELLE}

Voltaire est l'un des nombreux auteurs qui ont développé la conception de la religion naturelle au siècle des Lumières ${ }^{19}$, aux côtés de Jean-Jacques Rousseau dans La profession de foi du vicaire savoyard (1762) ou Denis Diderot dans De la suffisance de la religion naturelle (1747 / publié en 1770). L'idée de religion naturelle jalonne toute l'œuvre de Voltaire. Elle est présente, pour ne citer que quelques titres, dans les Éléments de la philosophie de Newton (1738), « Le souper » du chapitre XII de Zadig (1748), le Poème sur la loi naturelle (1756) ou encore le Dictionnaire philosophique, qui connaît plusieurs éditions à partir de 1764 (Dictionnaire philosophique portatif) jusqu'à 1785 (édition posthume de $\mathrm{Kehl}^{20}$ ), en passant par l'édition définitive de 1769 (La Raison par alphabet). Le Dictionnaire philosophique voit la naissance de son projet à la cour de Frédéric II de Prusse, mais il ne sera réalisé qu'une dizaine d'années plus tard, dans l'atmosphère de scandale qui entoure les affaires Calas et Sirven, pris en défense par Voltaire, puis celle du chevalier de la Barre, que le Dictionnaire de Voltaire va accompagner dans les flammes du bûcher en 1766.

Le dessein de Voltaire, qui a auparavant participé à la rédaction du Dictionnaire de l'Académie, puis à l'Encyclopédie de Diderot et d'Alembert, est d'offrir dans son Dictionnaire une somme de ses idées en matière de philosophie, de société, de politique et de religion, en les mettant à la portée du plus grand nombre de gens - ce qu'il estime difficilement faisable avec la publication volumineuse et

${ }^{19}$ Aussi bien sur le plan philosophique que littéraire. Sur ce sujet voir surtout : G. Gusdorf, Dieu, la nature, l'homme au siècle des Lumières, Payot, Paris 1972, pp. 122-142. Raymond Trousson dresse une longue liste des auteurs d'utopie à l'époque des Lumières qui incluent la religion naturelle dans leurs fictions : R. Trousson, Religions d'utopie, Éditions Ousia, Bruxelles 2001, pp. 163-189. Trousson constate : "Sous une forme ou une autre s'impose ainsi un déisme rationaliste. Les découvertes scientifiques, la connaissance de lois générales ne permettent plus guère de retenir miracles et prophéties comme preuves de la toute puissance de Dieu. Ces miracles, même, ne contrediraient-ils pas plutôt la sagesse divine? Fondée sur la raison universelle, la religion naturelle se donne pour la seule possible. Si le déisme peut admettre que l'adoration d'un Dieu se manifeste dans un culte public, les religions particulières apparaissent comme des institutions nécessaires, mais seulement d'un point de vue politique » (ibidem, p. 169).

${ }^{20}$ Cette édition, outre les articles du Dictionnaire philosophique, comprend aussi ceux, nombreux, des Questions sur l'Encyclopédie publiés entre 1770 et 1772, les articles de Voltaire rédigés pour l'Encyclopédie de Diderot et d'Alembert, ainsi que pour le Dictionnaire de l'Académie française. 
coûteuse de l'Encyclopédie. Il utilise à cette fin la forme du dictionnaire portatif, un type d'ouvrage maniable qui jouit d'une énorme popularité au XVIII ${ }^{\mathrm{e}}$ siècle. Les éditions successives du Dictionnaire philosophique, dont la diffusion rapide inquiète les autorités, vont provoquer une vague de condamnations, à commencer par celle du Parlement de Paris et celle du Siège-Siège, qui met le livre à l'Index ${ }^{21}$ en 1765, peu après sa publication. De nombreux ouvrages polémiques voient le jour : ceux de Louis-Mayeul Chaudon, Aimé-Henri Paulian, Laurent François ou Claude-François Nonnotte. Tous les pourfendeurs de Voltaire cités sont des ecclésiastiques dévoués à la tâche de l'apologie du catholicisme, celui-ci constituant l'une des cibles privilégiées de la pensée critique des Lumières.

Voltaire est sans doute le représentant le plus accompli de cette pensée, qu'il articule inlassablement durant une cinquantaine d'années. Omniprésente dans l'œuvre voltairienne, la religion ${ }^{22} \mathrm{~s}^{\prime} \mathrm{y}$ trouve le plus souvent associée à la superstition et au fanatisme - marques de l' " infâme » que Voltaire ne cesse d'appeler à écraser par la diffusion des idées de raison, de liberté et de tolérance. Légère, satirique, ironique ou sarcastique, la critique à laquelle il soumet la religion est cependant loin d'être irréfléchie ou gratuite. Visant à détruire et éradiquer ce qu'il y a de superstitieux ou d'absurde dans la religion, critiquant impitoyablement les abus qu'elle génère, Voltaire n'en tente pas moins de saisir ce qui constitue le noyau de la religiosité, et qu'il assimile à la religion naturelle ${ }^{23}$. Cette dernière peut se résumer en quelques points : 1) elle admet l'existence d'un Être suprême créateur (ou formateur) de l'univers, assimilé traditionnellement au Grand Horloger ou au Grand Architecte ; 2) elle considère cet Être comme prévoyant, juste et parfait, sans le définir ou formuler de dogmes à son sujet ; 3) elle correspond à la morale universelle ou loi naturelle, admise par tous les hommes en tout lieu, inscrite par Dieu dans le cœur humain ; 4) elle se résume dans l'exercice du culte intérieur, de la justice et de la charité, ou dans le respect de ce qu'on nomme la Règle d'or. Dans les premières éditions du Dictionnaire philosophique, l'article « Religion » est composé de « Sept questions sur la religion » (l'édition de 1769 contient une question en plus), dont la cinquième fournit un bref exposé de la religion naturelle :

${ }^{21}$ Afin de mieux cerner le contexte, voir : L. Macé, « Les Lumières françaises au tribunal de 1'Index et du Saint-Office », [dans :] Christianisme et Lumières, S. Albertan-Coppola, A. McKenna (dir.), Presses universitaires de France, Paris 2003, pp. 13-25. Macé écrit dans sa conclusion : « La question du style, récurrente dans les censures de Voltaire, ne doit-elle pas être lue comme l'indice d'une inquiétude croissante face à un phénomène nouveau propre aux Lumières : l'émergence progressive d'un public élargi, susceptible d'entrer aisément en contact avec les doctrines des philosophes? L'ironie n'était assurément pas à mettre entre toutes les mains » (ibidem, p. 24-25).

22 Sur ce sujet, voir notamment : B. Baczko, Job, mon ami : promesses du bonheur et fatalité du mal, Gallimard, Paris 1997, chapitre I ; B. Cottret, Le Christ des Lumières : Jésus de Newton à Voltaire, Éditions du Cerf, Paris 1990 ; R. Pomeau, La religion de Voltaire, Nizet, Paris 1956.

${ }^{23}$ Sur la religion naturelle voltairienne, voir : J. Lagrée, op. cit., pp. 62-67. Selon Lagrée, chez Voltaire, " la religion naturelle apparait selon les textes tantôt comme la religion universelle des simples, tantôt comme la religion épurée des philosophes » (ibidem, p. 65). 
Après notre sainte Religion, qui sans doute est la seule bonne, quelle serait la moins mauvaise ? Ne serait-ce pas la plus simple? Ne serait-ce pas celle qui enseignerait beaucoup de morale et très peu de dogmes ? celle qui tendrait à rendre les hommes justes, sans les rendre absurdes ? [...] celle qui n'enseignerait que l'adoration d'un Dieu, la justice, la tolérance et l'humanité ? ${ }^{24}$

\section{LE DICTIONNAIRE ANTI-PHILOSOPHIQUE DE CHAUDON}

Dans la préface de son Dictionnaire anti-philosophique polémique, le bénédictin Louis-Mayeul Chaudon justifie son entreprise par les progrès de l'impiété et la mode du temps. Il se résigne à la forme dictionnairique contre son gré, préférant sans doute un discours structuré à la « raison par alphabet»:

On a mis l'erreur en Dictionnaire, il est nécessaire d'y mettre la vérité. Les Apôtres de l'impiété prennent toutes sortes de formes pour répandre leur poison, les Défenseurs de la Religion ne chercheront-ils pas aussi les moyens de faire goûter leurs remedes ? L'ordre alphabétique est le goût du jour, et il faut bien s'y plier si l'on veut avoir des Lecteurs ${ }^{25}$.

Chaudon connaît bien son adversaire : malgré les esquives et les démentis de Voltaire, il attribue le Dictionnaire philosophique à l'auteur de la Pucelle d'Orléans, reconnaissant dans l'ouvrage fatal " cette plume téméraire et féconde, que le Démon de l'esprit et de l'irréligion a préparée lui-même dans les gouffres de l'Enfer $»^{26}$. L'auteur du Dictionnaire anti-philosophique, qui s'appuie dans son livre sur un certain curé de Brie, dont le Traité abrégé de la religion est dispersé sur les pages du Journal Chrétien, résume ainsi le contenu du dictionnaire voltairien : « Ce Livre brise tous les liens qui attachent les hommes à la vertu. A peine reconnoit-il l'existence de l'Etre Supreme, et cette existence, de la façon qu'il l'admet, n'intéresse en aucune façon les hommes $»^{27}$.

Ce qui ressort du texte est son caractère tranché, son jugement intransigeant et univoque porté sur le Dictionnaire, ne laissant quasiment aucune place à un débat quelconque. Le scepticisme voltairien, toujours nuancé et évitant tout dogmatisme dans les questions métaphysiques ou morales, se trouve réduit ici à l'expression la plus scandaleuse de l'impiété, de l'irréligion et d'un athéisme matérialiste déguisé. Chaudon ne semble pas tenir compte du fait que Voltaire, en se réclamant du

${ }^{24}$ Voltaire, Dictionnaire philosophique, portatif, Londres 1764, <http://gallica.bnf.fr/ark:/12148/ btv1b8626129s $>$ [consulté le 29.06.2015], p. 318. Voltaire associe la religion naturelle à la sagesse chinoise dans l'article « Catéchisme chinois » (ibidem), pp. 91-116. Nous en trouvons aussi une autre illustration condensée dans l'article «Théiste » du Dictionnaire philosophique (édition de 1769).

25 L.-M. Chaudon, Dictionnaire anti-philosophique, pour servir de commentaire et de correctif au Dictionnaire philosophique, et aux autres livres, qui ont paru de nos jours contre le christianisme, chez la veuve Girard \& François Seguin, Avignon 1767, <http:/gallica.bnf.fr/ark:/12148/bpt6k6545729z> [consulté le 29.06.2015], p. V. Une version complétée de l'ouvrage en deux volumes intitulée Anti-dictionnaire philosophique paraît en 1775.

26 Ibidem, p. VI.

${ }^{27}$ Ibidem, pp. V-VI. 
théisme, s'oppose aussi bien à l'athéisme qu'au panthéisme. En fait, à mi-chemin entre le théisme et le déisme, Voltaire est déiste contre les théistes « naïfs » (enclins à admettre trop facilement la possibilité d'une révélation ou l'immortalité de l'âme) et théiste « pratique » contre les déistes pratiquement athées ${ }^{28}$.

Le Dictionnaire anti-philosophique de Chaudon contient aussi l'arrêt du Parlement condamnant le livre de Voltaire à être « lacéré et brûlé » par l'exécuteur de Haute-Justice, précisant que «Si l'Auteur étoit connu, il ne vous paroîtroit pas moins digne que son Ouvrage des peines les plus rigoureuses $»^{29}$. En justifiant la condamnation, l'arrêt affirme notamment :

On paroît admettre une Religion naturelle dans laquelle on reconnoîtroit un Dieu quelconque ; mais quelle seroit cette Religion, et quel Dieu y reconnoîtroit-on, puisque, selon l'Auteur, on n'a aucune idée de Dieu, qu'on ne peut le connoître, et qu'on ne lui rendroit aucun culte, sous prétexte qu'il n'a pas besoin de nous?

Mysteres, Dogmes, Morale, Discipline, Culte, Vérité de la Religion, Autorité divine et humaine, tout est donc en butte à la plume sacrilége de cet Auteur qui se fait gloire de se ranger dans la classe des bêtes en mettant l'homme à leur niveau, puisqu'il n'admet de bonheur que celui des sens, et qu'il consent à périr entièrement comme elles ${ }^{30}$.

Chaudon adhère supposément sans restriction à la cette condamnation, qui impute à l'auteur du Dictionnaire philosophique portatif le matérialisme le plus exacerbé. La religion naturelle, évoquée dans l'arrêt, s'y trouve rabaissée au niveau de création purement abstraite et stérile, car privée d'une idée de Dieu et d'un culte concrétisés. Chaudon reprend la même critique de la religion naturelle dans l'article « Religion », en insistant sur son inefficacité, son insuffisance et ses aberrations, propos que Voltaire retournerait sans doute volontiers contre la religion révélée :

La Religion naturelle nous donne, il est vrai, certains principes ; mais nous fournit-elle des motifs efficaces pour combattre nos contradictions et pour remplir nos devoirs ? Offre-t-elle des remedes à nos maux, des ressources à nos chûtes, des objets à nos desirs et à nos besoins ? Quelle récompense assure-t-elle à la vertu ? Quelle punition au vice ? Les plus sages Philosophes eurent quelques notions de Dieu, de l'homme et de ses devoirs. Mais dans ce peu de lumieres, que de ténebres et d'extravagances! Leur science n'aboutit qu'à les rendre vains, superstitieux, idolâtres. L'homme a donc besoin d'un nouveau flambeau qui éclaire et dirige mieux sa raison. Jusqu'ici elle a été insuffisante ; et les hommes n'ont fait que l'obscurcir par leurs erreurs et leurs vices ${ }^{31}$.

Pour Chaudon - qui semble confondre quelque peu la religion naturelle et les religions païennes (qui se situent nécessairement pour lui sur le plan « naturel ») - les superstitions, les erreurs, les indécences et les cruautés des religions de toutes nations confondues s'opposent manifestement à la religion révélée professée par les Hébreux, dont le christianisme constitue la continuation logique et

\footnotetext{
28 J. Lagrée, op. cit., pp. 62-67.

${ }^{29}$ L.-M. Chaudon, op. cit., p. XVI.

30 Ibidem, pp. XVI-XVII.

31 Ibidem, p. 297.
} 
légitime ${ }^{32}$. Paradoxalement, dans la religion révélée de Chaudon, assimilée à la "Religion primordiale » transmise aux premiers hommes, tout paraît en même temps naturel et divin, alors que les religions païennes des idolâtres ont précisément dénaturé cette pensée divine originelle.

La remarque est intéressante dans la mesure où Voltaire reproche exactement la même chose aux religions révélées, qui ont selon lui dénaturé la simplicité et la pureté de la religion naturelle, non plus révélée certes (du moins dans le sens historique du terme), mais inscrite par Dieu dans la nature des choses. Pour Voltaire, l'incompréhensibilité et l'absurdité des mystères, des dogmes et des miracles, les contradictions des Écritures et des prophéties, ne font qu'obscurcir ce qu'il y a d'essentiel, de bienfaisant et d'utile dans la religion, sont source de fanatisme et d'intolérance. Par ailleurs, les ressemblances entre les religions mettent en doute le caractère exclusif du christianisme et renvoient au dénominateur commun de tous les cultes, auquel il s'agit d'accéder pour rendre la religion socialement valable.

\section{LE DICTIONNAIRE PHILOSOPHO-THÉOLOGIQUE DE PAULIAN}

L'attitude adoptée par le jésuite Aimé-Henri Paulian dans son Dictionnaire philosopho-théologique portatif de 1770 ne diffère pas en principe de celle de Chaudon. Selon lui, le Dictionnaire philosophique de Voltaire, condamné à juste titre, n'est qu' " un amas informe de faussetés, d'impiétés, de blasphêmes et d'obscénités $»^{33}$. Tout comme Chaudon d'ailleurs, Paulian choisit le dictionnaire comme forme d'expression pour des raisons sociales. Son ouvrage, dédicacé au Dauphin (futur Louis XVI), se veut être en effet un traité philosopho-théologique dirigé contre les esprits forts et les philosophes modernes. Certaines personnes avaient d'ailleurs déconseillé à Paulian le recours à l'ordre alphabétique et lui avaient suggéré une forme plus systématique. «Mais puisque dans ce siecle rien n'est plus à la mode que les Dictionnaires - constate-t-il dans la préface — et que trois fameux Dictionnaires ont presque fait tout le mal que nous déplorons, je les ai ramenées sans peine à ma maniere de penser $»^{34}$.

Paulian, dans l'article « Religion » de son dictionnaire, discute successivement les problèmes de la religion naturelle et de la religion révélée : judaïque, chrétienne considérée en général (christianisme) puis considérée en particulier (catholicisme). Dans ses réflexions sur la « Religion purement naturelle », il semble mieux que

32 Ibidem, p. 299.

33 A.-H. Paulian, Dictionnaire philosopho-théologique portatif, contenant l'accord de la véritable philosophie avec la saine théologie, et la réfutation des faux principes établis dans les écrits de nos philosophes modernes, Gaude, Nîmes 1770, <http://gallica.bnf.fr/ark:/12148/bpt6k6544328v> [consulté le 29.06.2015], p. II.

34 Ibidem, p. IV. 
Chaudon tenir compte des idées réelles de Voltaire. Il les présente non sans ironie, imaginant le discours qu' aurait pu selon lui faire un partisan de la religion naturelle :

C'est une Religion qui rejettant toute révélation divine, ne propose que des vérités conformes aux lumieres de la droite raison. Une personne qui professe une pareille Religion, avouera sans peine qu'un Etre infiniment parfait a tiré du néant ce vaste univers, qu'il le conferve et qu'il le gouverne par les loix les plus sages et les plus équitables : il ajoutera que l'homme ne sauroit trop lui prodiguer ses adorations, le remercier des biens dont sa main libérale l'a comblé et le comble tous les jours, et appaiser sa colere, lorsque par malice ou par fragilité il a eu le malheur de l'offenser : il fera même, si l'on veut, des leçons pathétiques de la plus saine morale $[\ldots]^{35}$.

La conclusion de Paulian, qu'on devine au style railleur du propos, est celle de l'insuffisance de la religion naturelle « de droit et de fait », concession faite toutefois que la dite religion est «bonne et nécessaire [...] que les préceptes qu'elle fait, sont justes et indispensables $»^{36}$. Ce jugement mitigé sur la religion naturelle - d'une part elle constitue le fondement rationnel de toute religion, de l'autre elle est insuffisante car réduisant la religion au niveau de la nature - semble préoccuper l'auteur du Dictionnaire philosopho-théologique, qui a besoin de recourir à l'éventualité d'un argument purement autoritaire fondé sur la souveraineté divine : « Mais enfin quand même (ce qu'il est impossible de prouver) la Religion révélée ne nous seroit pas absolument nécessaire, il suffit que le Maître suprême ait voulu l'ajouter aux lumieres de la raison, pour que nous soyons obligés de l'embrasser ${ }^{37}$. Et Paulian de reprendre la même pensée dans l'article sur la «Révélation »: «A-t-il plu au souverain Maître d'ajouter une Religion révélée aux lumieres de la raison? C'est un de ces faits qu'on ne discute pas par des raisonnements [...] $»^{38}$.

Cette argumentation qu'on pourrait taxer d' « absolutiste », essentiellement anti-rationaliste, étouffant dans l'œuf toute possibilité de rendre la religion naturelle suffisante, se trouve toutefois complétée et équilibrée chez Paulian par la conviction profonde de l'unité du dessein de Dieu et du caractère progressif de sa réalisation :

La Religion Romaine est sans contredit la plus ancienne Religion du monde. Elle subsiste depuis Adam jusqu'à nous, puisqu'elle observe la loi naturelle, la loi écrite en ce qu'elle a d'essentiel, je veux dire, en ce qui regarde le Décalogue et le Messie promis à l'univers, et la loi chrétienne ou évangélique. Oui, la loi naturelle, la loi écrite et la loi chrétienne, ne font qu'une feule et même Religion, émanée de Dieu dès l'origine du monde ${ }^{39}$.

La conception d'une religion universelle, issue de la révélation primitive donnée à Adam, qui se développe dans le temps et trouve son accomplissement dans la venue du Christ et dans le catholicisme, a de tout temps été formulée au sein l'Église, notamment par Augustin dans ses Rétractations (I, XII, 3). Sa présence sous la plume d'un apologète du XVIII' siècle n'a donc pas de quoi nous surprendre. L'insuffisance

\footnotetext{
35 Ibidem, p. 271.

36 Ibidem, p. 272.

37 Ibidem, p. 274.

38 Ibidem, p. 290.

${ }^{39}$ Ibidem, pp. 285-286.
} 
de la religion naturelle selon Paulian est due à son imperfection et au stade où se trouvait l'humanité lorsqu'elle la professait. Cela suppose que la religion adamique, dégénérée suite au péché originel en religion naturelle, exige une restauration par le moyen d'une intervention directe du Créateur. La religion naturelle se situe là dans un tout autre contexte que dans la perspective voltairienne, où elle possède un caractère universel et inaltérable, et où les limitations de la nature ne sont pas perçues comme l'effet d'une faute ancestrale posant la nécessité d'une révélation.

\section{OBSERVATIONS CRITIQUES DE LAURENT FRANÇOIS}

L'ouvrage du lazariste Laurent François Observations sur la "Philosophie de l'histoire" et le "Dictionnaire philosophique" (1770) nous éclaire davantage sur les enjeux de la polémique concernant la religion naturelle. Dans La philosophie de l'histoire, publiée en 1765 sous le nom de l'abbé Bazin, œuvre fondatrice d'une réflexion sur l'histoire de l'humanité indépendante de la théologie, Voltaire revient dans le chapitre sur « La Religion des premiers hommes » à la « Deuxième question sur la religion » du Dictionnaire philosophique portatif publié l'année précédente. Il y reformule l'idée selon laquelle les premiers hommes, mus par l'instinct et confrontés aux dangers de la nature, ont commencé par adorer un seul dieu - chaque peuple le sien : "Lorsqu'après un grand nombre de siecles quelques sociétés se furent établies, il est à croire qu'il y eut quelque Religion, quelque espece de culte grossier $»^{40}$. Ce culte monolâtre, admettant l'existence d'autres dieux, s'est naturellement transformé en polythéisme, tandis que le monothéisme, «fruit de la raison cultivée, ou de la révélation » ${ }^{41}$, ne s'est développé que progressivement à l'issue de la réflexion de sages.

Les observations critiques de François sur cette thèse reviennent à dire que la religion des premiers hommes leur a été révélée par le Créateur, et que ses principes ont été obscurcis à partir de l'époque des descendants de Noé42. C'est pour la même raison que, ne pouvant accepter l'idée de Voltaire et de Warburton suivant laquelle les Juifs ne connaissaient pas l'immortalité de l'âme (« Première question sur la religion » du Dictionnaire ${ }^{43}$ ), François se réfère de nouveau à la conception de la Religion primitive, unique et universelle, révélée à l'humanité à l'aube de la création, et qui comprenait déjà le dogme de l'immortalité :

Le Peuple Juif ne pouvant ignorer le dogme d'une vie future, il n'étoit pas nécessaire que Moïse lui

${ }^{40}$ La philosophie de l'histoire, par feu l'abbé Bazin, Changuion, Amsterdam 1765, <http:// gallica.bnf.fr/ark:/12148/bpt6k57060758> [consulté le 30.06.2015], p. 14.

41 Ibidem, p. 15.

${ }^{42}$ L. François, Observations sur la "Philosophie de l'histoire" et le "Dictionnaire philosophique” avec des réponses à plusieurs difficultés, 2 vol., Pillot, Paris 1770, t. 1, <http://gallica.bnf. fr/ark:/12148/bpt6k6535835r> [consulté le 29.06.2015], pp. 13-20.

${ }^{43}$ Voltaire, Dictionnaire..., pp. 308-309. 
rappellât en termes clairs ce dogme : mais il falloit pour fonder une bonne Religion, qu'il lui rappellât la Religion primitive de l'homme, méconnue des Nations de son temps ; et qu'il lui donnât des Loix dont l'observation pût lui rendre salutaire le dogme d'une vie future. Voilà ce qu'a fait Moïse : ce n'est point une nouvelle Religion qu'il établit, ni une Religion qui dût être abolie : c'est la Religion de tous les temps et pour tous les lieux $[\ldots]^{44}$.

Il est clair que Voltaire, niant en principe la possibilité d'une révélation, attribue à la religion une origine purement naturelle, considérant le récit de la Genèse sur le drame du jardin d'Éden comme une fable et regardant l'enfance de l'humanité sous un angle foncièrement naturaliste ${ }^{45}$. Or, tous ceux qui attaquent sa philosophie sont convaincus de la réalité d'une révélation historique faite au commencement du monde, obscurcie par le péché originel puis renouvelée, allant d'Adam jusqu'à Jésus-Christ, en passant par Noé, Abraham, Moïse et les Prophètes. La tradition de cette révélation se perpétue et s'exprime dans l'enseignement de l'Église catholique, notamment dans ses dogmes.

François, en critiquant la "Cinquième question sur la religion » du Dictionnaire philosophique, où son auteur s'interroge sur « la moins mauvaise des religions ", observe : " Notre Religion ne sauroit être sainte et bonne sans être divine ; par conséquent sans être aussi ancienne que l'homme, dont elle est le premier devoir essentiel. Toutes les autres Religions ne sauroient donc être que mauvaises ${ }^{46}$. Si Voltaire avait médité le commandement de l'amour de Dieu et du prochain, ajoute-t-il, il y aurait trouvé toute la doctrine de l'Église. Pourtant, Voltaire a beau avoir médité ce double précepte - ainsi que la Règle d'or, qu'il mentionne déjà dans les Éléments de la philosophie de Newton en 1738, et qu'il faut associer au commandement de l'amour - il ne voit pas la nécessité ni même le besoin d'en tirer un dogme quelconque, le précepte se suffisant à lui-même, tout comme la religion naturelle.

\section{LE DICTIONNAIRE PHILOSOPHIQUE DE LA RELIGION DE NONNOTTE}

L'ouvrage de Claude-François Nonnotte ${ }^{47}$ voit le jour en 1772, alors que la polémique du jésuite autour de l'Essai sur les mœurs de Voltaire, initiée dans

${ }^{44}$ L. François, op. cit., t. 2, <http://gallica.bnf.fr/ark:/12148/bpt6k6535864g> [consulté le 29.06.2015], p. 111.

${ }^{45}$ Sur la désacralisation du Paradis au siècle des Lumières, voir : B. Baczko, op. cit., pp. 93-113.

46 Ibidem, p. 130.

${ }^{47}$ C.-F. Nonnotte, Dictionnaire philosophique de la religion, 4 vol., 1772, t. 1, <http://gallica. bnf.fr/ark:/12148/bpt6k9618383b> [consulté le 29.03.2016]. 
les années '50, n'est pas encore terminée ${ }^{48}$. Nonnotte, aspirant en bon jésuite à utiliser tous les moyens disponibles Ad maiorem Dei gloriam, est le seul des polémistes catholiques cités dans la présente étude, qui assigne à la forme moderne du dictionnaire une valeur positive, censée faciliter la réception et garantir une lecture attrayante :

La forme de Dictionnaire qu'on a donné à l'Ouvrage a paru la plus propre à faire un service prompt, à contenter l'avidité \& la curiosité du Lecteur, à prévenir l'ennui \& le dégoût, à fournir sur le champ dans le besoin, les éclaircissemens sur les faits, la décision sur les points qui seroient contestés, la résolution de tous les doutes. La plûpart des articles peuvent être regardés comme autant de petits Traités Philosophiques, où chaque sujet est présenté avec l'ordre, l'analyse, \& la clarté nécessaire pour contenter, instruire \& convaincre.

L'avertissement présente l'ordre suggéré de la lecture des articles, qui quoique autonomes, ne doivent pas être perçus comme détachés les uns des autres, mais formant un tout cohérent. Le lecteur devrait commencer, en toute logique, par l'article " Religion », enchaîner avec celui sur la "Révélation », avant de passer à ceux consacrés aux preuves fournies par les «Prophéties » et les « Miracles », à l'article sur l'«Écriture-Sainte » (son caractère authentique et divin), aux réflexions concernant la « Foi » et sa « certitude inébranlable », et aboutir aux textes sur les richesses du «Christianisme », l'« Évangile » et les « Conseils Évangéliques » ${ }^{49}$. C'est ainsi que se dessine le plan de la première partie de l'ouvrage. La seconde porte sur les questions épineuses des rapports entre la foi et la raison (elle comprend les articles " Raison », " Mystères », " Trinité », " Création », « Origine du Mal»), la troisième sur les questions que les philosophes aiment aborder pour discréditer la religion et propager le libertinage (" Matière », " Ame ", « Certitude », « Destin », etc. $)^{50}$, la quatrième est censée augmenter le respect et l'attachement pour la religion, « en reconnoissant toutes les absurdités, extravagances $\&$ fureurs dans lesquelles donnent ceux qui l'attaquent $»^{51}$. Y serviront les articles « Tolérance », « Persécutions », « Fanatisme » ou « Superstition ${ }^{52}$.

Dans les articles « Religion » et « Révélation » de son dictionnaire, l'abbé Nonnotte s'évertue à démontrer l'insuffisance de la religion naturelle et la nécessité d'une révélation. Il combat les arguments des philosophes visant à prouver le contraire, en prenant pour cibles principales Jacques-André Naigeon (co-auteur du Militaire philosophe de 1768), le vicomte Bolingbroke et Jean-Jacques Rousseau (les deux entretiens opposant le Déiste au Philosophe chrétien constituent une

48 C.-F. Nonnotte, Examen critique, ou Réfutation du Livre des mœeurs, Chez la Veuve Bordelet, Paris 1757, <http://gallica.bnf.fr/ark:/12148/bpt6k109231p> [consulté le 29.03.2016]. Nonnotte est aussi l'auteur des Erreurs de Voltaire, autre ouvrage réfutant les idées du philosophe; idem, Erreurs de Voltaire. Nouvelle édition, augmentée d'un $3^{e}$ volume intitulé : l'Esprit de Voltaire dans ses écrits, 3 vol., <http://gallica.bnf.fr/ark:/12148/bpt6k6313306n> [consulté le 29.03.2016].

49 C.-F. Nonnotte, Dictionnaire philosophique..., pp. III-VI.

50 Ibidem, pp. VI-VIII.

51 Ibidem, p. IX.

${ }^{52}$ Ibidem, pp. VIII-X. 
réplique à la Profession de foi du vicaire savoyard, qui en reprend littéralement le texte en l'attribuant au Déiste ; pp. 206-242). Il ne cite Voltaire que de façon occasionnelle, le nommant pourtant « chef des Apôtres » de la religion naturelle. Il lui reproche notamment le manque de clarté et l'indétermination de ses idées sur la loi naturelle, en lui opposant celles de Cicéron :

Il nous dit bien dans ses Mélanges, que le Déisme est la Religion du bon sens ; dans son Poëme sur la Religion Naturelle, qu'il suffit d'être juste, et que le reste est arbitraire ; avec le Bolingbroke, qu'il faut adorer Dieu, \& être honnête homme, \&c. Mais il se gardera bien de vous dire avec Cicéron, que la Loi naturelle est une lumiere que Dieu met dans les ames, pour leur faire découvrir les premieres vérités, connoître les premiers devoirs discerner, ce qui est juste de ce qui ne l'est pas ${ }^{53}$.

Tout en reconnaissant " la sainteté, la vérité et la nécessité » de la religion naturelle, Nonnotte la juge insuffisante pour deux raisons : premièrement, elle ne fournit pas de réponses à certaines questions essentielles concernant la condition humaine (péché originel, rédemption, vie future); deuxièmemement, elle échoue à remédier aux dérèglements de la nature humaine ${ }^{54}$. Comme le résume le Troisième et le Quatrième fait constatés dans l'article « Révélation » : «Les lumières des Sages n'ont pas été suffisantes pour dissiper les ténébres du genre humain, ni leurs efforts pour en arrêter les déréglemens » ${ }^{55} ;$ « Ce n'est que par la Révélation Chrétienne que les ténèbres ont été dissipées, et que les déréglemens ont été arrêtés $\|^{56}$. Par ailleurs, nous retrouvons chez Nonnotte l'argumentation « absolutiste » de Paulian : il suffit que l'Être suprême ait souhaité révéler des vérités supérieures à l'homme, pour que celui-ci, ayant reconnu le Créateur, soit obligé de les embrasser. Or, la révélation s'imposant à lui par la force de l'évidence, la subtilité de ses dogmes et la perfection de sa morale, l'homme, doté de raison et de libre arbitre, doit s'y soumettre. Pour Nonnotte, comme pour les autres apologistes cités précédemment, le catholicisme est la religion universelle de l'humanité, révélée à celle-ci à Adam après la Chute. En témoignent les pratiques sacrificielles ou la sanctification du septième jour, connus des nations païennes depuis le commencement du monde et annonçant respectivement le sacrifice du Sauveur et le sabbat, préfiguration du jour de sa résurrection ${ }^{57}$.

Nous n'allons plus nous étendre sur l'argumentation voltairienne, prenant le contre-pied de celle de l'abbé Nonnotte : la révélation, au lieu de dissiper les ténèbres de l'humanité, n'a fait que les amplifier, elle n'a en rien remédié aux vices des hommes et en a suscité de nouveaux. Quant aux ressemblances entre les traditions païennes et le christianisme, elles ne témoignent nullement en faveur de

${ }^{53}$ C.-F. Nonnotte, Dictionnaire philosophique..., t. 4, <http://gallica.bnf.fr/ark:/12148/bpt6k96338639> [consulté le 6.06.2016], p. 96.

54 Ibidem, p. 25.

55 Ibidem, p. 147.

56 Ibidem, p. 158.

57 Ibidem, pp. 38-43. 
celui-ci. Elles renvoient plutôt soit à un même goût pour la superstition, soit au dénominateur commun, pur et inaltérable, de la religion naturelle.

\section{CONCLUSION}

Le débat sur la religion universelle — naturelle pour Voltaire et révélée pour ses détracteurs - rappelle un dialogue de sourds, les partis opposés partant de principes différents et arrivant nécessairement à des conclusions différentes. Au Moyen Âge, les ancêtres des dictionnaires et des encyclopédies - les sommes, les specula, les compilations étymologiques - étaient enracinés dans une conception métaphysique et théologique. Les dictionnaires thématiques du siècle des Lumières, champ de conflit idéologique, ne sont pas moins ancrés dans la philosophie ou la théologie, mais désormais, suivant une logique plurielle et polémique issue du déchirement entre les partisans de la tradition et ceux du progrès. Le dictionnaire est signe d'appartenance à un milieu, représentatif et porteur d'une certaine vision du monde, dont la religion demeure un élément-clef. Chaudon se lamentait en 1767 en parlant du Dictionnaire philosophique portatif:

Ce Dictionnaire n'est point une de ces futilités littéraires, qui de la toilette d'un petit Maître passent dans la boutique d'un Epicier. Tout le monde le lit; tout le monde le cite ; Militaires , Magistrats, Femmes, Abbés, c'est une coupe, dans laquelle tous les états et tous les âges s'abreuvent du poison de l'impiétés8.

Il s'agit là de l'enjeu majeur du conflit herméneutique qui oppose à l'époque catholicisme dogmatique conservateur et philosophie rationaliste moderne. Celleci s'est vu frayer le chemin de la conscience collective depuis près d'un siècle et les Églises traditionnelles sont sur la défensive dans leur lutte pour les âmes. Pour les tenants d'un catholicisme qui sert d'appui à un pouvoir politique absolutiste, il s'agit alors de mobiliser les forces et de resserrer les rangs face aux « ennemis de la foi », quitte à confondre des opinions qui souvent n'ont pas grand-chose en commun : libertins, philosophes, hérétiques, sociniens, déistes, athées, se trouvent tous mis dans le même sac. Pourtant, les divergences entre ces différents groupes de penseurs, unifiés il est vrai dans une attitude critique vis-à-vis des anciennes autorités, sont considérables. Voltaire ne s'entend que partiellement, voire mal, avec Rousseau et les pasteurs genevois ${ }^{59}$, il est en guerre contre d'Holbach et le « quatrième imposteur », l'athée militant ou le Militaire philosophe. Le catholicisme lui aussi est loin de former un tout compact et homogène. Nombreux sont au sein du clergé les partisans des Lumières, qui expriment ouvertement leurs idées, hésitent ou les dissimulent, à l'instar du curé Meslier. Le rationalisme ronge l'Église de l'intérieur à partir du XVII ${ }^{\mathrm{e}}$ siècle, et il ne faut pas oublier que les

58 L.-M. Chaudon, op. cit., p. V.

${ }^{59}$ Voir sur ce sujet : R. Pomeau, op. cit., pp. 193-201, 280-303, 336-344. 
jésuites ont joué un rôle non négligeable dans la formation de l'idée de religion naturelle, dont Voltaire, leur ancien élève, se fera l'apôtre ${ }^{60}$.

Mais cette complexité et ces ambiguïtés caractérisant le champ des idées à l'aube de la modernité s'efface derrières les évidences du conflit idéologique, et derrière les positions tranchées qu'il génère et impose à ses participants. L'enjeu est en effet de taille. Car les nouvelles idées circulent et se propagent en premier lieu dans les cercles d'intellectuels, de savants, de philosophes ou d'illuminés et n'atteignent que rarement les couches inférieures et populaires de la sociétét ${ }^{61}$. Or, les ouvrages de vulgarisation que constituent les dictionnaires, comme celui, portatif, de Voltaire, s'avèrent être une arme redoutable, sapant les anciens fondements et disloquant au sein du peuple les repères traditionnels, à commencer par la Révélation, opposant l'idée d'une religion universelle naturelle à celle, admise de tout temps, qui conditionnait la vision du monde et de l'Ancien Régime. On connait le rôle que jouera cette idée de la religion naturelle au temps de la Révolution et à sa suite : le culte de la Raison oscillant entre déisme et athéisme, le culte de l'Être Suprême d'inspiration rousseauiste, et la théophilanthropie d'inspiration voltairienne ${ }^{62}$, vont se relayer avant de céder la place à la « religion de la majorité des Français » concordataire. Ici de nouveau, la complexité de la situation religieuse, illustrée par une Église déchirée entre prêtres jureurs et réfractaires, sera reléguée au second plan par la violence aveugle de la Terreur, qu'elle soit blanche ou jacobine. Le conflit idéologique mené dans les dictionnaires de la seconde moitié du siècle se verra transporté dans les rues et sur les champs de bataille, où s'affronteront révolutionnaires et réactionnaires, républicains et royalistes.

Les dictionnaires thématiques, qui ont eu un impact crucial sur la société française (surtout masculine) du XVIII ${ }^{\mathrm{e}}$ siècle, accélérant la révolution des idées et des mœurs qui marque l'époque, continuent d'être de véritables machines de guerre au XIX ${ }^{\mathrm{e}}$ siècle, dans le contexte de la crise moderniste touchant l'Église. Ainsi,

${ }^{60}$ Ibidem, pp. 46-64. C'est notamment l'activité missionnaire des jésuites en Chine (ou en Nouvelle-France, comme le montre l'œuvre de Joseph-François Lafitau) qui a poussé certains d'eux (Louis Lecomte, René-Joseph de Tournemine) à adopter l'idée de religion naturelle, quitte à relativiser l'importance de la révélation judéo-chrétienne et du péché originel, ou bien à accentuer l'accès à la révélation (dès l'origine du monde) de toute l'humanité.

${ }^{61}$ G. Gusdorf, op. cit., pp. 35-38.

${ }^{62}$ R. Pomeau, op. cit., pp. 451-455. Au sujet des cultes révolutionnaires, voir surtout : M. Vovelle, 1793, La Révolution contre l'Eglise. De la Raison à l'Etre suprême, Éditions Complexe, Bruxelles 1988 ; A. Aulard, Le culte de la raison et le culte de l'Etre suprême (1793-1794). Essai historique, Félix Alcan, Paris 1892, <http://classiques.uqac.ca/classiques/aulard_alphonse/culte_ de_la_raison_et_etre_supreme/culte_de_la_raison.html> [consulté le 29.03.2016] ; A. Mathiez, Les Origines des cultes révolutionnaires (1789-1792), Librairie G. Bellais, Paris 1904, <http://gallica. bnf.fr/ark:/12148/bpt6k206888> [consulté le 1.04.2016] ; La Théophilanthropie et le culte décadaire, 1796-1801. Essai sur l'histoire religieuse de la Révolution, Félix Alcan, Paris 1903, <http:// gallica.bnf.fr/ark:/12148/bpt6k118370z> [consulté le 1.04.2016]. 
du côté catholique, l'abbé Jacques Paul Migne se distinguera par la publication de plusieurs séries de la monumentale Encyclopédie théologique (1844-1866), où la conception du catholicisme comme religion universelle, issue d'une révélation primitive et dispersée dans tous les cultes du monde, occupe une place de choix. Les dictionnaires apologétiques combattront les thèses formulées dans les milieux libéraux, républicains et anticléricaux, héritiers de la pensée voltairienne, qui donneront naissance à des ouvrages comme l'Encyclopédie nouvelle (1834-1841) de Pierre Leroux et Jean Reynaud, ou le Dictionnaire Universel (1854-1856), puis le Nouveau dictionnaire universel (1865-1870) de Maurice Lachâtre. Mais cela est bien sûr matière à une autre étude.

\section{THE DICTIONARY AS AN IDEOLOGICAL BATTLEFIELD: NATURAL RELIGION AND UNIVERSAL RELIGION IDEAS IN THE DICTIONARIES OF THE AGE OF ENLIGHTENMENT (1764-1775)}

\section{Summary}

The article is a reflection on the dictionary as an ideological battleground illustrated with the example of thematic dictionaries or encyclopedias of the Age of Enlightenment. The perspective of the study is philosophical and religious, centred on the ideas of natural and universal religion as elements of conflicting worldviews. We will discuss the controversy surrounding the Philosophical Dictionary by Voltaire, who was attacked by Catholic clergymen such as Chaudon, Paulian, François or Nonnotte.

Key words: dictionary, encyclopedia, philosophy, natural religion, universal religion, ideological conflict, worldview, Voltaire. 